\title{
De la idea al proyecto de ESCUELA 2.0
}

\section{Enric Torres Barchino ${ }^{a}$, Manuel Martínez Torán ${ }^{b}$ y Manuel Contero González}

${ }^{\mathrm{a}} \mathrm{I} 3 \mathrm{~B}$, Universitat Politècnica de València, entorbar@ega.upv.es, ${ }^{\mathbf{b}}$ Departamento de Dibujo, Universitat Politècnica de València, mmtoran@upv.es y ${ }^{\mathbf{c}} \mathrm{I} 3 \mathrm{~B}$, Universitat Politècnica de València, mcontero@upv.es

\begin{abstract}
Spain is the second country in the EU (28) with more school drop-outs. $18.5 \%$ of students do not complete secondary school and is the fourth country in the OECD (35) with 23.2\% of young people (16-29 years) that neither study nor work. In recent years, the educational movement STEM or STEAM is presented as an alternative to learning with an extensive background and recognized around the world. In our country a political debate with vision for the future and the participation of society and the educational community is necessary. Reflect on the current educational model and how implement it will help that he is more equitable and quality. Despite this situation, and although unequally, (academic) formal and non-formal education (makers spaces, do-it-yourself, slow-education, etc.) move towards innovation and inclusion of people and virtual environments of learning. The information and communication technologies (ICT) and the methodologies based on projects (PBL), the use of personal learning environments (PLE) learning and networking (PLN), are generating great expectations in the education sector. The decided educational bet from institutions and programmes for training and evaluate the teaching staff can improve the competences and skills that students should acquire to give meaning to the so-called school 2.0. In this context, we will try to answer the following questions: If there is more technology in the classroom, you will get more knowledge? With the e-learning is improved the motivation of learning to learn? Are we losing the skills of the know-how? Why is innovation in education important?, and What do we mean by innovative school?
\end{abstract}

Keywords: educational path, teaching teams, school 2.0, STEM+, training of teachers, PBL, hyper class and analysis of experiences of innovation. 


\section{Resumen}

España es el segundo país de la UE(28) con más abandono escolar, el $18,5 \%$ de alumnos no terminan la ESO y es el cuarto de la OCDE(35) con el 23,2\% de jóvenes (16-29 años) que ni estudian ni trabajan. En los últimos años, el movimiento educativo STEM o STEAM se presenta como una alternativa de integración de aprendizajes, además, cuenta con una amplia experiencia y de reconocimiento en todo el mundo. En nuestro pais hace falta un debate politico con visión de futuro, con la participación de la sociedad y de la comunidad educativa. Reflexionar sobre el actual modelo educativo y de cómo implementarlo ayudará a que sea más equitativo y de calidad. Pese a esta situación, y aunque de manera desigual, tanto la educación formal (académica), como la no formal (makers spaces, do-it-yourself, slow-education, etc.) se mueven hacia la innovación e inclusión de las personas y hacia entornos virtuales de aprendizaje. Las tecnologías de la información y de la comunicación (TIC), junto con las metodologías basadas en proyectos (PBL), el uso de entornos personales de aprendizaje (PLE) y de trabajo en red (PLN), están generando grandes expectativas en el sector educativo. La apuesta decidida de las instituciones educativas y de programas para la formación y evaluación del profesorado, pueden mejorar las competencias y habilidades que deben adquirir los alumnos para dar sentido a la denominada Escuela 2.0. En este contexto, trataremos de dar respuesta a las siguientes preguntas: ¿a más tecnología en el aula, se adquiere mayor conocimiento?, con el aprendizaje virtual (e-Learning) ¿se mejora la motivación por el aprender a aprender?, ¿se están perdiendo las habilidades del saber hacer?, ¿por qué es importante la innovación en educación?, y ¿qué entendemos por escuela innovadora?

Palabras clave: Senda educativa, equipos docentes, escuela 2.0, STEM+, formación del profesorado, PBL, hiperaula, análisis de casos de innovación.

\section{Introducción}

La generalización en el uso de las TIC en la Escuela, está suponiendo sobre todo adquirir habilidades para el uso de ordenadores, aplicaciones para los teléfonos inteligentes, tabletas, pizarras digitales, o para comunicar informaciones online. un gran reto para los métodos de enseñanza del profesorado y para el aprendizaje colaborativo de los estudiantes. 
La denominada Escuela 2.0 está siendo la palanca del cambio educativo, su objetivo es poner en marcha las aulas digitales del siglo XXI, aulas dotadas de infraestructura tecnológica y de conectividad, para garantizar un cambio en la metodología de trabajo, ya que en la actualidad disponemos de aulas del siglo XIX, alumnos del siglo XXI y profesores del siglo XX.

El aprendizaje virtual y en red, junto con las propuestas que en la actualidad se están realizando desde la educación formal, como desde la educación no formal (makers spaces, MOOCs, do-it-yourself, after-school, eLearning, etc.), están haciendo desaparecer las barreras espacio-temporales. Además, la formación es cada vez más flexible, y el profesorado pasa a ser un tutor que orienta y facilita los procesos formativos, cuya comunicación de contenidos es constante y actualizada.

Las nuevas metodologías de aprendizaje, como por ejemplo las basadas en proyectos o problemas $(\mathrm{PBL})^{1}$, en entornos personales de aprendizaje (PLE) ${ }^{2}$ y en las redes personales de aprendizaje $(\mathrm{PLN})^{3}$, están suponiendo una demanda creciente en la denominada sociedad del conocimiento y de la innovación.

En España, en los últimos años se están mejorando los resultados académicos como la tasa de idoneidad ${ }^{4}$, abandono escolar ${ }^{5}$ y fracaso escolar ${ }^{6}$, así como la adquisición de competencias $^{7}$ y habilidades que deben adquirir los alumnos. Aún así, estos índices siguen siendo altos, en 2017, el 18,5\% de alumnos no terminaron la Educación Secundaria Obligatoria (ESO), mientras que en la UE(28) fue del 11\%.

${ }^{1}$ Project Based Learning (PBL). Disponible: https://es.wikipedia.org/wiki/M\%C3\%A9todo de proyectos

${ }^{2}$ Personal Learning Environment (PLE). Disponible: https://es.wikipedia.org/wiki/Entorno personal de aprendizaje

${ }^{3}$ Personal Learning Network (PLN). Disponible: https://es.wikipedia.org/wiki/Red_personal_de_aprendizaje

${ }^{4}$ Tasa de idoneidad: porcentaje de alumnos que realiza un curso escolar según su edad. Por ejemplo, en $1^{\circ}$ curso ESO el $86,10 \%$ pasa de curso (13,90\% repite curso), mientras que en $3^{\circ}$ curso ESO el $67,50 \%$ pasa de curso $(32,50 \%$ repite curso).

5 Abandono escolar: en España, el 19\% de la población entre 18-24 años no logra titularse en Bachillerato o Formación Profesional.

${ }^{6}$ Fracaso escolar: alumnos que a los 16 años no logran el Graduado en la ESO. En 2017, el 18,50\% no logró el Graduado en la ESO.

${ }^{7}$ Competencias: el proyecto DeSeCo (OCDE, 2003) definió el concepto de competencia como "la capacidad de responder a demandas complejas y llevar a cabo tareas diversas de forma adecuada". 
La senda educativa (itinerarios de formación que siguen los jóvenes a partir de los 16 años), es del 66\%, mientras que el $34 \%$ no sigue ningún tipo de estudios.

En el curso 2015-16, la población escolar (enseñanzas no universitarias) fue de 8.108.679 alumnos, distribuyéndose así: educación infantil, primaria y ed.especial 4.766.071 alumnos, Educación Secundaria Obligatoria 1.868.584 alumnos, Bachillerato 694.224, FP-Básica 62.025, FP-GM 350.226, FP-GS 355.277, y otros programas 12.272 alumnos.

La población de jóvenes (a partir de 16 años) que continuaban su formación postobligatoria, fue de 1.461.752 alumnos, distribuyéndose así: FP-Básica 62.025 (4,24\%), FP-GM 350.226 (23,95\%), FP-GS 355.277 (24,30\%), y Bachillerato 694.224 (47,49\%).

Por otra parte, la pérdida de matrícula en Ingeniería sigue siendo preocupante, dada la necesidad de empleo tecnológico que requiere una economía basada en la innovación. Sigue la tendencia de los últimos años, las mujeres en Ingeniería representan el 23,6\% y los hombres el 76,4\%, y los titulados universitarios vinculados a estudios STEM ${ }^{8}$ representan el $26 \%$.

En este contexto, las metodologías basadas en proyectos o problemas (PBL), el aprendizaje virtual (eLearning) y la integración de áreas STEM están adquiriendo gran protagonismo, junto con las metodologías activas ${ }^{9}$, pero todas estas técnicas y tecnologías, ¿están mejorando significativamente el aprendizaje de conocimientos y habilidades?, o más bien, ¿son usadas mayoritariamente para el entretenimiento?

Las administraciones educativas (MECD y CC.AA.) están haciendo esfuerzos para desarrollar planes de formación del profesorado y para la innovación docente. Es cierto, que el voluntarismo es el factor que determina que los centros educativos asuman como una oportunidad incluir las TIC y el cambio metodológico que supone la Escuela 2.0, pese a que la educación en España aún sigue sin un rumbo claro. Se pierde mucho talento joven, y la mucha dedicación del profesorado se diluye y no se obtienen los resultados esperados.

Existen múltiples factores que influyen en el desinterés y la desmotivación por el aprendizaje, entre otras razones por las trabas que supone el conocimiento estanco de las asignaturas del currículo. Se sigue suspendiendo mucho. Por ejemplo, en $1^{\circ}$ curso ESO (1213 años) promocionan el $83,7 \%$ de chicos y el $89 \%$ de chicas, mientras que en $4^{\circ}$ curso ESO (15-16 años) es del 80,7\% de chicos y del $87,9 \%$ de chicas.

${ }^{8}$ El concepto STEM / STEAM (Science, Technology, Engineering, Art and Math) fue concebido por John Maeda (EE.UU, Seattle, 1966) siendo presidente de la Rhode Island School of Design (EE.UU, Rhode Island). Disponible: https://www.risd.edu/

${ }^{9}$ Metodologías activas. Disponible: http://blogbibliotecas.mecd.gob.es/2015/10/27/nuevos-modelosde-ensenanza-libros-recientes-en-la-biblioteca/ 
Sin embargo, cuando analizamos cual es el porcentaje de alumnos con todas las asignaturas aprobadas (superadas a final de curso), la situación cambia. En $1^{\circ}$ curso ESO es del 51,5\% de chicos, y del $64 \%$ de chicas, mientras que en $4^{\circ}$ curso ESO, pasa a ser del $61,7 \%$ de chicos y del $71,4 \%$ de chicas.

La edición de PISA-2015 (evaluación de los jóvenes de 44 países de la OCDE sobre qué saben y son capaces de hacer a los 15 años), se centra en el rendimiento en matemáticas, ciencias y comprensión lectora. España se sitúa en una posición intermedia respecto de la OCDE(44). En Matemáticas se sitúa en la posición 28, en Lectura en la posición 24, y en Ciencias en la posición 27. Ahora bien, respecto de la UE(32), España se sitúa en Matemáticas en la posición 27, en Lectura en la posición 22, y en Ciencias en la posición 25. Entre 2012 y 2015, 7 de 10 países con los mejores resultados en matemáticas, ciencias y comprensión lectora fueron asiáticos.

La investigación que se presenta, analiza el contexto educativo (ESO y Bachillerato), y cómo las nuevas metodologías se pueden implementar en el proceso de enseñanza y aprendizaje, a través de la propuesta de integrar áreas de conocimiento STEM.

\section{El concepto de Escuela 2.0 y la necesidad de innovar en educación}

Diversos autores ${ }^{\mathbf{1 0}}$ e instituciones educativas ${ }^{11}$ hace tiempo que están desarrollando experiencias sobre la denominada Escuela 2.0. Los puntos en común son: necesidad de compartir los contenidos que se han creado, nuevos métodos más participativos, la comunidad educativa requiere tener capacidades y habilitades, metodologías y materiales para atender la diversidad del alumnado, así como generar y facilitar el acceso a materiales digitales educativos. Ya no es suficiente disponer de aulas digitales, software y formación en TIC.

La economía y la sociedad cambian cuando los factores de producción se combinan de una manera novedosa (Schumpeter, 1961). Schumpeter introdujo el concepto de innovación y desarrolló las siguientes ideas: la búsqueda a través de la investigación de nuevos conocimientos o soluciones suponen curiosidad y renovación, y las invenciones e innovaciones son la clave del crecimiento económico y social.

\footnotetext{
${ }^{10}$ Blog de Ángel Fidalgo. Escuela 2.0. es algo más que digitalización de contenidos, ordenadores y comunicaciones. Disponible: https:/innovacioneducativa.wordpress.com/2009/05/05/escuela20-es-algo-mas-que-digitalizacion-de-contenidos-ordenadores-y-comunicaciones/

${ }^{11}$ MECD-INTEF. Instituto Nacional de Tecnologías Educativas y de Formación del Profesorado. Disponible: http://educalab.es/intef/introduccion
} 
En este sentido, a continuación se identifican aquellos factores que influyen para que se produzca innovación en el contexto escolar ${ }^{12,13}$.

a) Factores que promueven la innovación educativa:

- Equipos docentes sólidos y comunidad educativa receptiva

- Redes de intercambio y cooperación (uso de TIC)

- Contexto social, compromiso y creatividad

- Institucionalización de la innovación

- Reflexión y evaluación

- Políticas de la Administración educativa y formación docente

b) Factores que dificultan la innovación educativa:

- $\quad$ Estructura escolar rígida y casi invariable desde el s.XIX

- Resistencia y rutinas del profesorado

- Individualismo y corporativismo

- Pesimismo y malestar docente

- Efectos perversos de las reformas y currículum fragmentado

- Divorcio entre teoría y práctica

Para promover innovación en el aula, hace falta conocer y aplicar cuantas más y diversas metodologías mejor. A veces, la innovación se produce por el solo hecho de estructurar las ideas, trabajar en equipo y tener en cuenta el contexto social, para que más tarde se concreten en acciones.

\section{3. ¿Dónde se está innovando y cuales son los resultados según PISA?}

Un aspecto importante de la investigación, es inventariar y documentar aquellas experiencias educativas más significativas que aporten innovación y buenas prácticas. En el libro editado por Fundación Telefónica ${ }^{14}$, recoge los testimonios de "gigantes" de la educación de todo el mundo ${ }^{15}$, realidades educativas en países y contextos muy distintos con el objetivo de liderar soluciones innovadoras para mejorar la educación.

\footnotetext{
${ }^{12}$ Estudio sobre innovación educativa en España, No17. (MECD, 2011). Disponible: https://sede.educacion.gob.es/publiventa/estudio-sobre-la-innovacion-educativa-enespana/educacion-espana/14970

13 Innovación educativa en España: un estudio descriptivo a partir de los datos contenidos en REDINED. Disponible: http://redined.mecd.gob.es/xmlui/handle/11162/86731

${ }^{14}$ Observatorio. Innovación para la inclusión. Disponible: https://observatorio.profuturo.education/blog/descarga/gigantes-de-la-educacion/

15 Red Educativa Mundial (REDEM). Disponible: http://www.redem.org/conozca-los-11-colegiosmas-innovadores-del-mundo/
} 
Para entender por qué algunos países avanzan hacia la equidad y la calidad educativa, los denominados informes PISA evaluan cada tres años, la comprensión lectora, matemática y de ciencias de los alumnos de 15 años. A partir del año 2018, PISA evaluará la competencia lectora en entorno digital, así como la capacidad de analizar asuntos globales e interculturales, cómo emprender acciones por el bien común y el desarrollo sostenible.

\begin{tabular}{|c|c|c|c|c|c|c|c|}
\hline $\begin{array}{c}4^{\circ} \text { ESO } \\
(15-16 \text { años) }\end{array}$ & España & $\begin{array}{c}\% \text { nivel } \\
\text { bajo }\end{array}$ & $\begin{array}{l}\text { UE } \\
(\mathbf{3 2})\end{array}$ & $\begin{array}{c}\% \text { nivel } \\
\text { bajo }\end{array}$ & $\begin{array}{c}\text { OCDE } \\
(44)\end{array}$ & $\begin{array}{c}\% \text { nivel } \\
\text { bajo }\end{array}$ & $\begin{array}{c}\text { PAISES } \\
\text { TOP }\end{array}$ \\
\hline Lectura & $\begin{array}{c}495,58 \\
(\mathbf{2 4} / \mathbf{4 4})\end{array}$ & $16 \%$ & 494,45 & $20 \%$ & 492,55 & $20 \%$ & $\begin{array}{r}\text { Singapur } 535,10 \\
\text { Canadá } 526,67 \\
\text { Finlandia } 526,42\end{array}$ \\
\hline Matemáticas & $\begin{array}{c}485,84 \\
(\mathbf{2 8} / 44)\end{array}$ & $22 \%$ & 492,64 & $22 \%$ & 490,20 & $23 \%$ & $\begin{array}{r}\text { Singapur 564,19 } \\
\text { Japón 532,44 } \\
\text { Corea Sur 524,11 }\end{array}$ \\
\hline Ciencias & $\begin{array}{c}492,79 \\
(27 / 44)\end{array}$ & $18 \%$ & 494,80 & $21 \%$ & 493,20 & $21 \%$ & $\begin{array}{r}\text { Singapur 555,57 } \\
\text { Japón 538,39 } \\
\text { Estonia } 534,19\end{array}$ \\
\hline
\end{tabular}

Tabla 1. Resultados PISA-2015 en Lectura, Matemáticas y Ciencias. (Fuente: Informe PISA-2015. MECD y elaboración propia)

\section{Hipótesis y objetivos de la investigación}

\subsection{Hipótesis}

Una hipótesis es una suposición de algo posible o imposible para sacar de ello una o más conclusiones, es una idea que puede no ser verdadera, pero que está basada en información previa. Su valor reside en la capacidad para establecer más relaciones entre los hechos y explicar por qué se producen. Una hipótesis científica es una proposición aceptable que ha sido formulada a través de la recolección de información y datos, aunque no esté confirmada, sirve para responder de forma alternativa a un problema con base científica.

Las hipótesis pueden generar predicciones, las cuales son declaraciones que proponen que una variable impulsará un cierto efecto o cambio en otra variable como resultado de un experimento controlado. 
Para formular una hipótesis tendremos en cuenta lo siguiente:

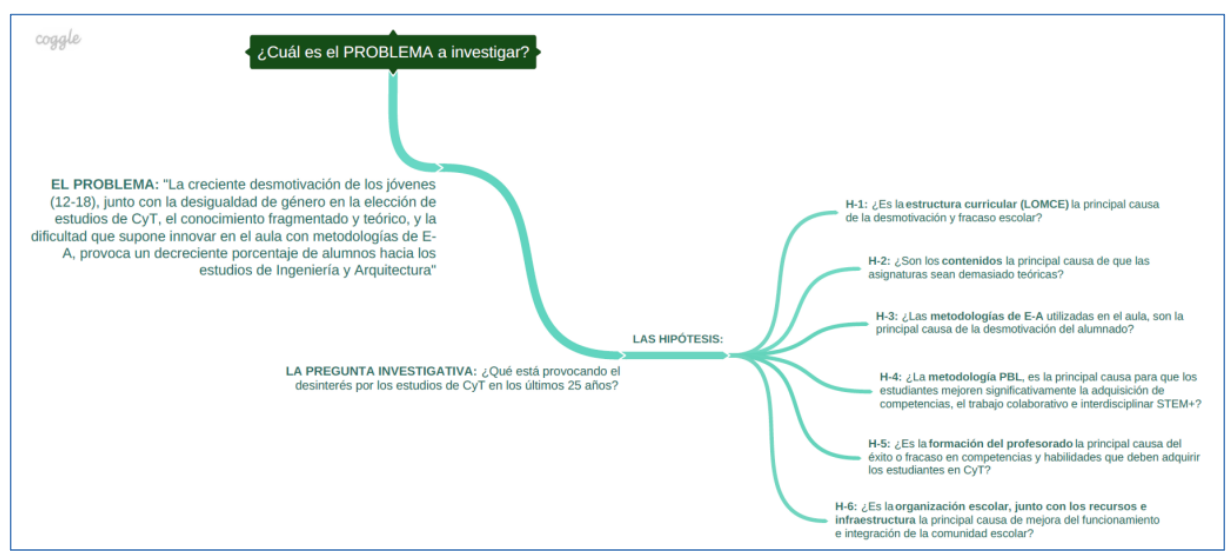

Fig. 1 Mapa mental: ¿Cuál es el problema a investigar? Definir hipótesis (Fuente: Elaboración propia)

\subsection{Objetivos}

Para definir un objetivo de investigación, se debe tener en cuenta el fin al que se desea llegar, la meta que se pretende lograr, y evitar en su redacción juicios y consideraciones personales. El objetivo de una investigación determina e influye en el marco teórico o la metodología a seguir, y se suelen redactar en infinitivo, deben ser claros, alcanzables y pertinentes, y estarán planteados a partir de un problema o una hipótesis.

Distinguiremos entre objetivos generales y específicos:

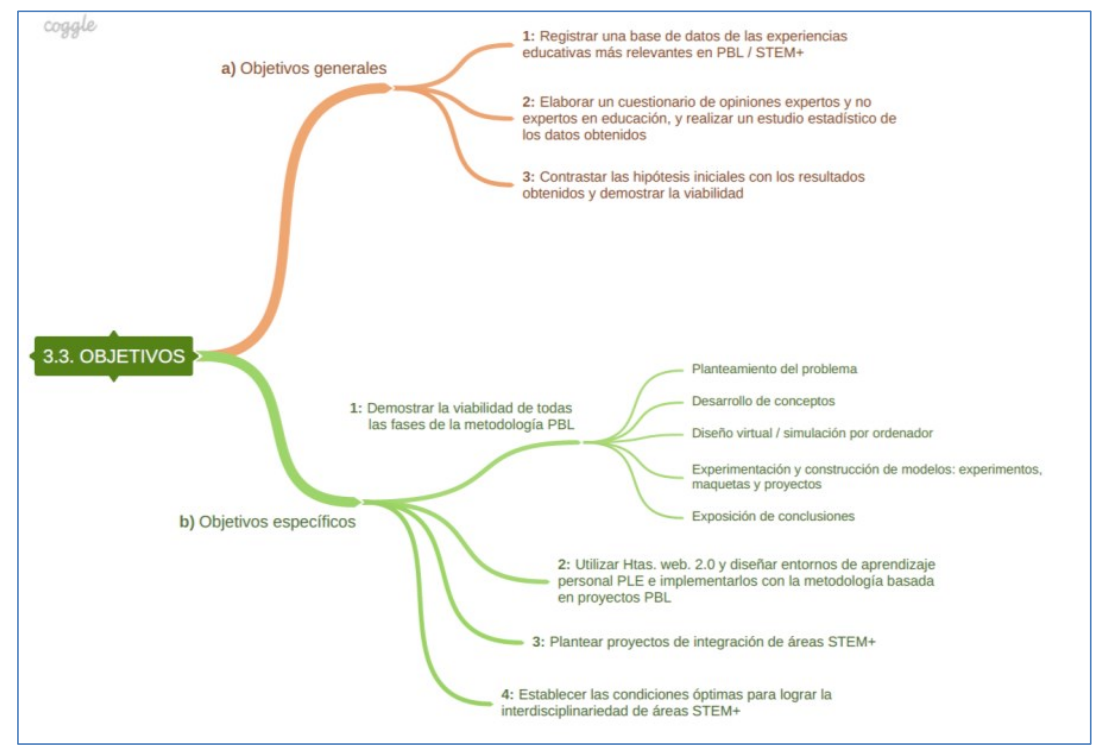

Fig. 2 Mapa mental: Definición de objetivos generales y específicos (Fuente: Elaboración propia) 


\section{Desarrollo de la investigación}

Para realizar la investigación, se seguirá el método científico tanto para la perspectiva cualitativa (entrevistas de opinión) como la cuantitativa (experimentos y encuestas). En la tabla 2, se resumen las tres fases (planificación, ejecución y resultados) de la investigación.

\begin{tabular}{|c|c|c|}
\hline FASE 1 & Planificación & 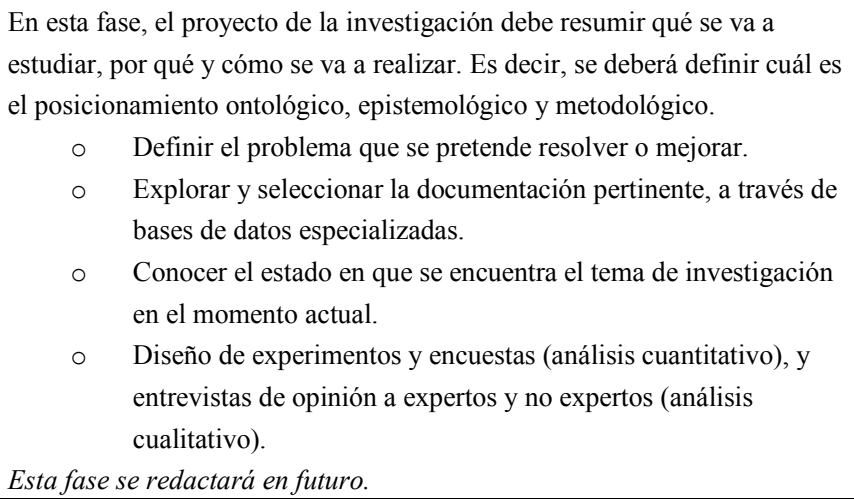 \\
\hline FASE 2 & Ejecución & $\begin{array}{l}\text { En esta fase, es el momento de desarrollar propiamente la investigación a } \\
\text { fin de lograr los objetivos propuestos. } \\
\begin{aligned} \text {. Imaginar una o varias respuestas probables, y deducir las } \\
\text { consecuencias de las hipótesis emitidas. }\end{aligned} \\
\circ \quad \text { Hacer operativos los objetivos y su desarrollo metodológico. } \\
\circ \quad \begin{array}{l}\text { Observar y contrastar la realidad en función de las hipótesis } \\
\text { planteadas, a través del trabajo de campo in situ. }\end{array} \\
\quad \quad \begin{array}{l}\text { Tratamiento de datos y resultados estadísticos (SPSS) y } \\
\text { cualitativos (ATLAS). }\end{array} \\
\text { Esta fase se redactará en presente. }\end{array}$ \\
\hline FASE 3 & Resultados & $\begin{array}{l}\text { En esta fase, se deben exponer los resultados de la investigación, y } \\
\text { elaborar un documento donde se describa el proceso de cómo se llegó a } \\
\text { dichos resultados. } \\
\circ \text { Analizar e interpretar los datos obtenidos, y establecer } \\
\text { conclusiones a través de los resultados de la investigación. } \\
\circ \quad \text { Redacción, preparación y presentación de la investigación. } \\
\circ \quad \text { Extender las conclusiones y establecer el marco adecuado para } \\
\text { incrementar el interés y la motivación por el conocimiento de } \\
\text { las tecnologías. } \\
\text { Esta fase se redactará en pasado. }\end{array}$ \\
\hline
\end{tabular}

Tabla 2. Fases de la investigación. (Fuente: elaboración propia)

Básicamente, la investigación pretende que los estudiantes (ESO y BCyT) sean capaces de diseñar su propio Entorno de Aprendizaje Personal (PLE), entorno tecnológico centrado en lo que hace el alumno y caracterizado por la flexibilidad para aprender mediante las TIC, de 
manera que les permita integrar tanto las tecnologías y herramientas, como los procesos y prácticas, puesto que el sujeto que aprende, explora, define y desarrolla cualidades metacognitivas adquiere las competencias digitales. Un PLE es algo así como el conjunto de software necesario para realizar las tareas de un proyecto. Existe infinidad de software, cada uno de estos programas se clasifican en función del tipo de tarea a realizar:
a) Estructurar ideas, crear y diseñar
b) Buscar información y aprender
c) Organizar y compartir
d) Comunicar, interactuar y publicar

Además, los estudiantes deben aprender a organizar su propia Red de Aprendizaje Personal (PLN), con el objetivo de implementar los conocimientos adquiridos a través del aprendizaje virtual y en redes colaborativas, para transformarla en una experiencia o proyecto tecnológico en el Aula-Taller. A través de una red PLN, los alumnos se mantienen en contacto e intercambian información. Diseñar un PLN, es la forma en que nos conectamos para ayudarnos a aprender, es un sistema que ayuda a tomar el control para gestionar el propio aprendizaje.

Por tanto, si los estudiantes son capaces de diseñar su PLE, además de saber gestionar su PLN, muy probablemente mejoraran la gestión del conocimiento y sabran compartir recursos. Obviamente, el objetivo de la investigación es demostrar la viabilidad que supone la realización de proyectos tecnológicos entre diversas materias del currículo, integrándolas en el proyecto STEM. A través de la metodología de proyectos PBL y de su realización física, los alumnos adquieren una visión global y una experiencia contrastada entre el aprendizaje virtual y el real-físico.

La utilización de un PLN sirve para:

- El desarrollo professional y seguimiento a expertos en un àrea de conocimientos

- Localizar recursos para el aula

- Obtener ideas para las clases y las programaciones didácticas

- Informarse sobre cómo integrar las TIC al proceso educativo

- Encontrar soluciones a diversas problemáticas de un modo colaborativo

- Mantenerse continuamente informado sobre la actualidad educativa

Poner en marcha un PLN significa actuar en diferentes ámbitos:

- Redes sociales (Facebook, Google+).

- Microblogging (Twitter).

- Perfiles profesionales (LinkedIn, Xing).

- Wikis (Wikispaces, PBWiki, Edublogs).

- Blogs (WordPress, Blogger).

- Real Simple Syndication (GoogleReader, NetVibes). 
- Nings (comunidad de personas interesadas en los mismos temas)

- Marcadores sociales ("tus favoritos", Diigo, Delicious).

- Webinars (conferencias de expertos TED, Classroom 2.0, Edudemic).

- Backchanneling (narración en tiempo real de un evento, caracterizado por un hashtag).

Por último, la investigación debe contrastar que la metodología $\mathrm{PBL}^{\mathbf{1 6}}$, es realmente una herramienta pedagógica que acerca al estudiante al mundo real, y en el caso concreto de la investigación, acerca al los estudiantes al mundo de la tecnología y de la técnica. Los alumnos de manera colaborativa deben saber resolver problemas tecnológicos de la vida cotidiana. La metodología PBL, si va acompañada de otras medidas organizativas a nivel escolar, consigue que los estudiantes cooperen y adquieran de manera eficaz conocimientos y habilidades. A través de la metodología PBL, se consigue motivar a los estudiantes por la CyT, además de tener una visión global y saber resolver los problemas planteados.

Analizar objetos y sistemas técnicos, diseñar, experimentar y desarrollar proyectos en el ámbito de la enseñanza de las tecnologías, amplía el nivel de conocimientos, además de mejorar significativamente la adquisición de competencias en creatividad y habilidades que requieren los estudiantes de 12-18 años.

Poner a prueba la investigación, supone demostrar la viabilidad de trabajar en el AulaTaller por proyectos y equipos interdisciplinares, para lo cual hay que elaborar encuestas de opinión, entrevistar y tomar datos con los usuarios, y obtener conclusiones cualitativas y cuantitativas. En definitiva, hay que valorar si fundamentalmente, la metodología PBL, junto con el trabajo colaborativo e interdisciplinar STEM, mejoran significativamente el aprendizaje tradicional.

Para que sea efectiva la interdisciplinariedad STEM, es necesario analizar los currículos de las diferentes asignaturas y niveles educativos, así como saber gestionar la complejidad que supone trabajar de manera colaborativa entre el profesorado y alumnado. Las fig. 3 y 4 muestran un ejemplo de interdisciplinariedad entre áreas de conocimiento afines a la Tecnología, de manera que pueda ser implementada por fases. El ejemplo sirve para la ESO como para la modalidad de CyT del Bachillerato.

\footnotetext{
${ }^{16}$ El aprendizaje basado en problemas o proyectos (Project Based Learning PBL), consiste en que un grupo de estudiantes de manera autónoma, aunque guiados por el profesor/a, deben encontrar la respuesta a una pregunta o solución a un problema. El PBL facilita la interdisciplinaridad y la integración de conocimientos, atravesando las barreras del conocimiento fragmentado de las disciplinas y materias. El PBL se sustenta en la teoría constructivista de Jean Piaget (1896-1980) y Lev Vygotski (1896-1934). Posteriormente fue desarrollado por W.H.Kilpatrick hacia 1918.
} 
De la idea al proyecto de Escuela 2.0

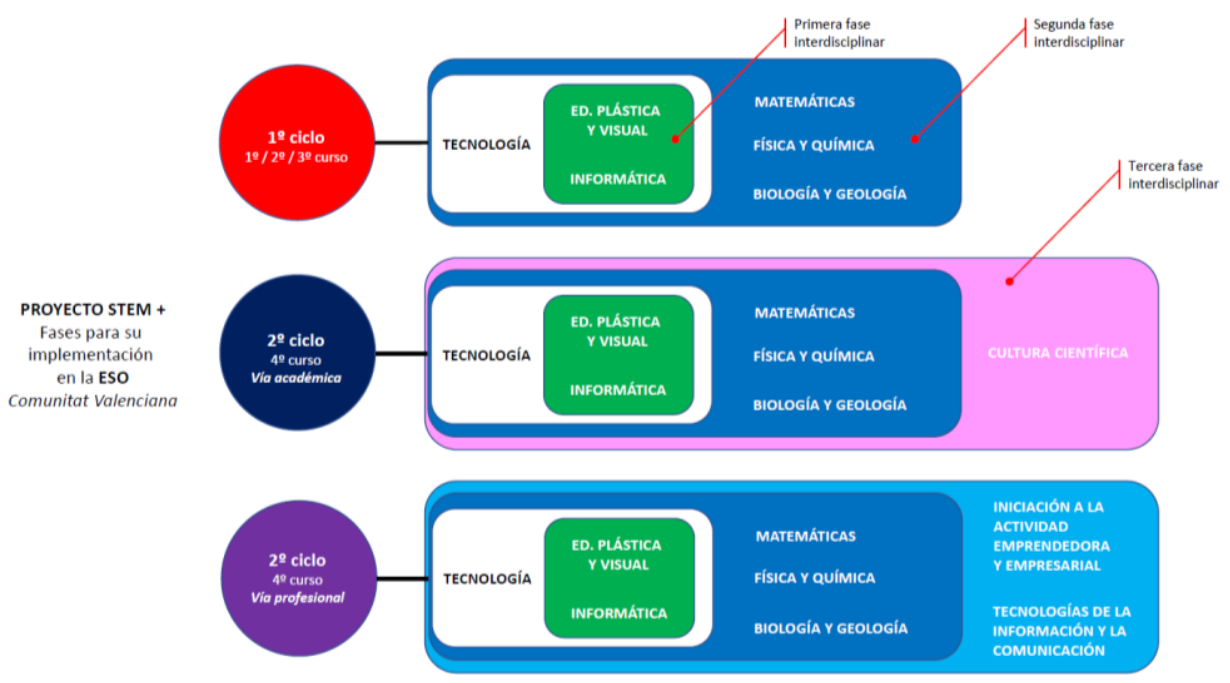

Fig. 3 Fases para la implementación de un proyecto STEM para la ESO (Fuente: Elaboración propia)

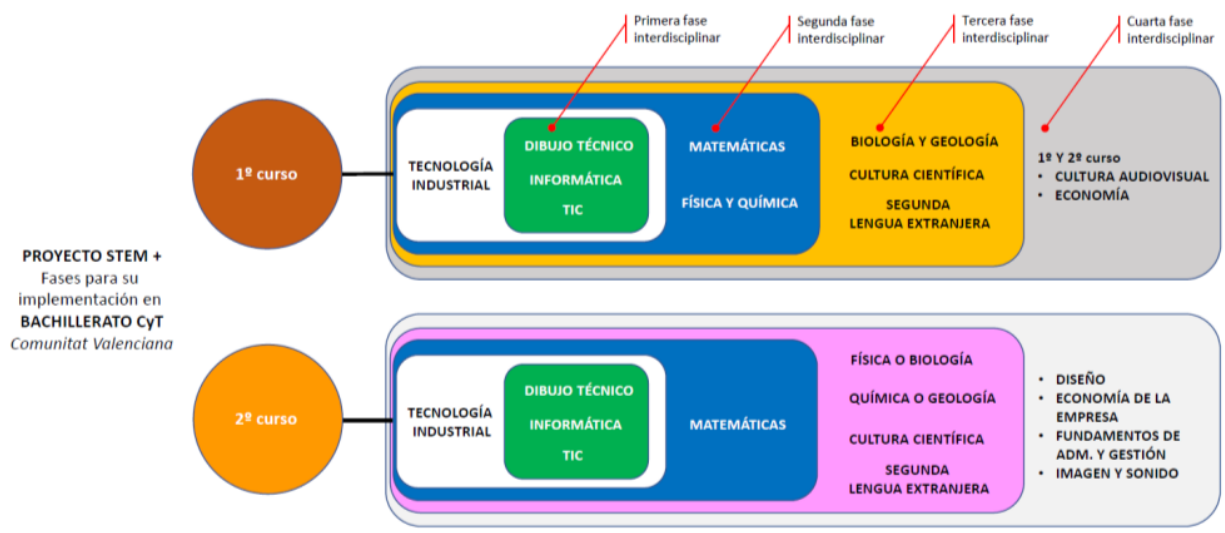

Fig. 4 Fases para la implementación de un proyecto STEM para BACHILLERATO (Fuente: Elaboración propia)

\section{Datos cuantitativos de la investigación}

Contrastar datos y elaborar conclusiones cuantitativas, implica realizar un "trabajo de campo". En 2018, los centros públicos de ESO y de BCyT son los indicados en la tabla 3.

\begin{tabular}{|c|c|c|}
\hline PROVINCIA & IES públicos - ESO & IES públicos - BCT \\
\hline Alacant & 136 & 129 \\
\hline Castelló & 49 & 40 \\
\hline València & 172 & 156 \\
\hline Total & 357 & 325 \\
\hline
\end{tabular}

Tabla. 3 Distribución de centros públicos por provincias de la C.Valenciana (Fuente: Elaboración propia) 
Para realizar el análisis cuantitativo (estadístico) es necesario determinar:

La muestra representativa (n) y el error muestral (e), que se calcula así:

Muestra (n): $n=\frac{N \cdot \sigma^{2} \cdot Z_{\alpha}^{2}}{e^{2} \cdot(N-1)+\sigma^{2} \cdot Z_{\alpha}^{2}} \quad$ Error muestral (e): $e=Z_{\alpha} \cdot \frac{\sigma}{\sqrt{N}} \cdot \sqrt{\frac{N-n}{N-1}}$

Según las tablas de distribución normal estándar, para un nivel de confianza $Z_{\alpha}$ del 95\%:

\begin{tabular}{|r|c|c|c|c|c|c|c|}
\hline Valor $Z_{\alpha}$ & 0,84 & 1,28 & 1,35 & 1,40 & 1,47 & 1,55 & 1,64 \\
\hline Nivel de confianza $Z_{\alpha}$ & $80 \%$ & $90 \%$ & $91 \%$ & $92 \%$ & $93 \%$ & $94 \%$ & $95 \%$ \\
\hline
\end{tabular}

Siendo:

\begin{tabular}{|c|l|c|}
\hline $\mathrm{N}$ & Tamaño de la población (centros públicos C.Valenciana) & 357 (ESO) + 325 (BCT) \\
\hline$\sigma$ & Desviación estándar & 0,5 \\
\hline$Z_{\alpha}$ & Valor del nivel de confianza & $95 \%$ \\
\hline$e$ & Límite aceptable de error & 0,05 \\
\hline
\end{tabular}

Según la formula anterior, para un nivel de confianza del $95 \%$ y un valor de $Z_{\alpha}$ de 1,64 , la muestra (n) a realizar por provincias será de:

\begin{tabular}{|c||c|c||c|c|}
\hline PROVINCIA & $\begin{array}{c}\text { Población }\left(\mathrm{N}_{1}\right) \\
\text { IES públicos } \\
\text { ESO }\end{array}$ & $\begin{array}{c}\text { Muestra }\left(\mathrm{n}_{1}\right) \\
\text { IES públicos } \\
\text { ESO }\end{array}$ & $\begin{array}{c}\text { Población }\left(\mathrm{N}_{2}\right) \\
\text { IES públicos } \\
\text { BCT }\end{array}$ & $\begin{array}{c}\text { Muestra }\left(\mathrm{n}_{2}\right) \\
\text { IES públicos } \\
\text { BCT }\end{array}$ \\
\hline Alacant & 136 & 90 & 129 & 86 \\
\hline Castelló & 49 & 41 & 40 & 35 \\
\hline València & 172 & 105 & 156 & 99 \\
\hline Total & 357 & $\begin{array}{c}236 \\
\text { centros a encuestar }\end{array}$ & 325 & $\begin{array}{c}220 \\
\text { centros a encuestar }\end{array}$ \\
\hline
\end{tabular}

Tabla. 4 Distribución de centros públicos por provincias de la C.Valenciana y muestra representativa

(Fuente: Elaboración propia)

Por tanto, para la obtención de resultados cuantitativos, hará falta obtener datos in situ a través del diseño de experimentos y su implementación con grupos de alumnos de "control" y de "contraste". De la misma manera, para la obtención de resultados cualitativos, se realizaran encuestas de opinión (profesorado y alumnado) y entrevistas a expertos y no expertos en educación de manera presencial y online.

Debido a la magnitud que supone la realización del "trabajo de campo", por tanto habrá que focalizar la investigación solo en la provincia de València (105 IES ESO y 99 IES BCT). 
Suponiendo que entre los meses de enero y mayo de 2019 se realicen las encuestas, y considerando una media de 25 alumnos del "grupo de control" y otros 25 alumnos del "grupo de contraste", y evaluando el $80 \%$ de los centros educativos de ESO, supondrá realizar 4.200 encuestas. De la misma manera, considerando una media de 20 alumnos del "grupo de control" y otros 20 alumnos del "grupo de contraste", al evaluar el 80\% de los IES de BCyT de la provincia de València, supondrá realizar 3.160 encuestas, tal y como se expone en las tablas 5 y 6.

\begin{tabular}{|c|c|c|c|c|c|}
\hline \multirow[b]{2}{*}{ PROVINCIA } & \multicolumn{3}{|c|}{$\begin{array}{c}\text { Muestra }\left(\mathrm{n}_{1}\right) \\
\text { IES públicos ESO }\end{array}$} & \multirow[b]{2}{*}{$\begin{array}{c}\text { Evaluar el } 100 \% \\
\text { supone realizar } \\
n^{0} \text { encuestas }\end{array}$} & \multirow[b]{2}{*}{$\begin{array}{c}\text { Evaluar el } 80 \% \\
\text { supone realizar } \\
\text { enero-mayo } 2019\end{array}$} \\
\hline & $\begin{array}{c}\text { Centros } \\
\text { ESO }\end{array}$ & $\begin{array}{c}\text { alumnos } \\
\text { grupo } \\
\text { control }\end{array}$ & $\begin{array}{c}\text { alumnos } \\
\text { grupo } \\
\text { contraste }\end{array}$ & & \\
\hline Alacant & 90 & 25 & 25 & $90 \times 50=4.500$ & --- \\
\hline Castelló & 41 & 25 & 25 & $41 \times 50=2.050$ & --- \\
\hline València & 105 & 25 & 25 & $105 \times 50=5.250$ & $84 \times 50=4.200$ \\
\hline & & & & $\begin{array}{c}\text { Total } \mathrm{n}^{\circ} \text { encuestas } \\
11.800\end{array}$ & $\begin{array}{c}\text { Total } n^{\circ} \text { encuestas } \\
4.200\end{array}$ \\
\hline
\end{tabular}

Tabla. 5 Relación del número de centros ESO por provincias, grupos de control y contraste, y total de encuestas a realizar en la provincia de València. (Fuente: Elaboración propia)

\begin{tabular}{|c|c|c|c|c|c|}
\hline \multirow[b]{2}{*}{ PROVINCIA } & \multicolumn{3}{|c|}{$\begin{array}{c}\text { Muestra }\left(\mathrm{n}_{2}\right) \\
\text { IES públicos } \mathrm{BCT}\end{array}$} & \multirow[b]{2}{*}{$\begin{array}{c}\text { Evaluar el } 100 \% \\
\text { supone realizar } \\
n^{\circ} \text { encuestas }\end{array}$} & \multirow[b]{2}{*}{$\begin{array}{c}\text { Evaluar el } 80 \% \\
\text { supone realizar } \\
\text { enero-mayo } 2019\end{array}$} \\
\hline & $\begin{array}{c}\text { Centros } \\
\text { BCT }\end{array}$ & $\begin{array}{c}\text { alumnos } \\
\text { grupo } \\
\text { control }\end{array}$ & $\begin{array}{l}\text { alumnos } \\
\text { grupo } \\
\text { contraste }\end{array}$ & & \\
\hline Alacant & 86 & 20 & 20 & $86 \times 40=3.440$ & --- \\
\hline Castelló & 35 & 20 & 20 & $35 \times 40=1.400$ & -- \\
\hline València & 99 & 20 & 20 & $99 \times 40=3.960$ & $79 \times 40=3.160$ \\
\hline & & & & $\begin{array}{c}\text { Total } \mathrm{n}^{\circ} \text { encuestas } \\
8.800\end{array}$ & $\begin{array}{c}\text { Total } n^{\circ} \text { encuestas } \\
3.160\end{array}$ \\
\hline
\end{tabular}

Tabla. 6 Relación del número de centros BCT por provincias, grupos de control y contraste, y total de encuestas a realizar en la provincia de València. (Fuente: Elaboración propia) 


\section{Conclusiones}

A través de las iniciativas llevadas a cabo por la Conselleria d'Educació, como por ejemplo, el Decreto 51/2018 (DOCV 8284, 27 abril) por el que se introducen nuevas materias, entre las que se destacan: "Talleres de Refuerzo", "Talleres de Profundización" y "Proyecto interdisciplinario" (trabajo por proyectos), así como la organización del "II Congreso Internacional STEM" (Burjassot, 9-10 nov. 2018), se prevé un incremento de proyectos de innovación en los centros educativos, que integren el aprendizaje virtual y el real-físico a través de la realización de experimentos, prototipos y maquetas $3 \mathrm{D}$, así como la inclusión de programas y actividades de carácter interdisciplinar STEM+.

Es importante que las instituciones educativas y la sociedad en general, tengan una mejor percepción social de la Formación Profesional y del actual Bachillerato de Ciencias, entre otras razones porque urge un incremento del número de estudiantes con formación STEM+, y que de continuidad a los estudios de Ingeniería y Arquitectura.

La puesta en marcha de esta investigación, abre la posibilidad de profundizar en aspectos de cómo los estudiantes adquieren conocimientos en red, así como el uso y la colaboración de herramientas web 2.0 (PLE y PLN). El uso de metodologías activas basadas en proyectos, implicará mayor motivación por el aprendizaje y evitará el abandono de tareas.

Paralelamente, es necesario realizar mejoras en la arquitectura de acondicionamiento y nuevos diseños para los espacios de aulas, talleres y laboratorios que incluyan instalaciones y uso de las TIC, delimitando los espacios para presentaciones (present, listen, share), interacción (interact, discuss, questions), de investigación (investigate, research, discover), de creación (create, imagine, explore), de intercambio (exchange, support, encourage), y de desarrollo (develop, plan, examine).

Los datos analizados sobre rendimiento académico y de motivación por el aprendizaje en la ESO, FP y Bachillerato, dejan un lastre de pérdida de talento en los jóvenes. El 34\% de los jóvenes a partir de los 16 años ( $\left.4^{\circ} \mathrm{ESO}\right)$ abandonan los estudios.

Existen varias incoherencias en la LOMCE (2013), sobre todo en $4^{\circ}$ curso ESO, pues los alumnos se dividen entre el itinerario de estudios de la vía académica (acceso a Bachillerato) y la vía profesional (acceso a Formación Profesional). La formación recibida por los alumnos de la vía profesional les impide acceder a Bachillerato, lo que supone entre otras cuestiones no lograr las competencias necesarias para alcanzar otros niveles de formación. Aunque cada vez hay más programas de compensación y diversidad curricular, acaban siendo poco eficientes para el excesivo esfuerzo que realiza el profesorado y la administración educativa.

Es preocupante que en 2017, la población en España de jóvenes de 16 años, el 18,5\% no terminaron la ESO, y que sólo el 66\% siguieron estudios a través de FP o de Bachillerato, 
es decir, el 34\% no sigue ningún tipo de estudios. Respecto de los alumnos que siguen estudios de FP-Básica es sólo del 4,24\%, mientras que los que acceden a FP-GM es del 23,95\%, FP-GS 24,30\%, y Bachillerato en sus tres modalidades el 47,49\%.

La pérdida de matrícula en Ingeniería es contínua, pese a la necesidad de empleo tecnológico que requiere una economía basada en la innovación. Las mujeres en Ingeniería representan el 23,6\% y los hombres el 76,4\%.

El horario escolar y el aprendizaje por asignaturas estancas del currículo, no favorece la motivación, ni la utilidad de lo que se estudia. La propuesta de integración de áreas STEM, facilitará el aprendizaje colaborativo entre asignaturas y profesorado, así como el cambio metodológico que supone aprender a resolver problemas reales a través de la realización de experimentos o proyectos, intercalando el aprendizaje virtual y el físico-real.

Se prevé que la toma de datos de la investigación cuantitativa y cualitativa se concluya hacia el mes de mayo de 2019, ya que la población (N) en la Comunidad Valenciana es elevada (357 IES ESO y 325 IES BCyT. Si se acepta que el nivel de confianza sea del 95\% y el límite de error del 0,05, ello supone que la muestra (n) sigue siendo muy alta, es decir, la muestra $\left(n_{1}\right)$ de IES ESO es de 236 y la muestra $\left(n_{2}\right)$ de IES de BCyT es de 220 centros a encuestar. Por este motivo, la investigación se centrará sólo en la provincia de València, bajando así el número de centros a encuestar: 105 centros ESO y 99 centros BCyT.

En definitiva, se pretende obtener datos del $80 \%$ de los centros educativos, a través de encuestas de opinión (grupos de control y de contraste) a través de 4.200 encuestas para IES ESO y de 3.160 encuestas para IES BCyT. El procesamiento de datos y obtención de resultados cuantitativos se realizará con el programa estadístico SPSS, y la obtención de resultados cualitativos se realizará con el programa ATLAS.

\section{Referencias}

FERNÁNDEZ ENGUITA, M. (2018). Más escuela y menos aula. Madrid: Morata.

HARGREAVES, A. (2003). Enseñar en la Sociedad del conocimiento. Barcelona: Octaedro.

MARINA, J.A. (2010). La educación del talento. Barcelona: Ariel.

ROBINSON, K. (2015). Escuelas creativas. Barcelona: Grijalbo.

SALINAS, J., PÉREZ, A. y BENITO, B. de. (2008). Metodologías centradas en el alumno para el aprendizaje en red. Madrid: Síntesis.

EL MUNDO. (2016). Así se enseña en el aula del futuro. Madrid: El Mundo. Disponible: https://www.elmundo.es/sociedad/2016/09/08/57d061c646163fd86d8b45cc.html 
GOSÁLVEZ, P. (2015). Los jesuitas revolucionan el aula. Lleida: El País. Disponible: https:/elpais.com/politica/2015/03/27/actualidad/1427473093_128987.html

INFORME PISA. (2015). Programa para la evaluación internacional de los alumnos. Informe español. Madrid: MECD. Disponible: https://www.mecd.gob.es/inee/evaluacionesinternacionales/pisa/pisa-2015.html

ROSENBERG, N. (2005). España va a sufrir mucho si no empieza a innovar. Madrid: El País.

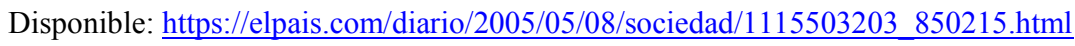

UNESCO. (2001). La enseñanza de las ciencias, la tecnología y las matemáticas en pro del desarrollo humano. Goa: Unesco-Castme. Disponible: https://es.scribd.com/document/142725393/Informe$\underline{\text { Unesco }}$

Blog de Ángel Fidalgo. Disponible: https://www.mindomo.com/es/mindmap/innovacion-educativa8af901 eead9a47a38ad22e3da45ade54

Buenas prácticas universitarias en innovación educativa. Disponible: http://138.4.83.162/organiza/buscador buenaspracticas/

Common framework for digital competence of teachers. Disponible: https://intef.es/blog/commonframework-for-digital-competence-of-teachers/

Consumerlab (2018). 10 Hot Consumer Trendes 2018. Disponible: https://www.ericsson.com/en/trends-and-insights/consumerlab/consumer-insights/reports/10-hotconsumer-trends-2018

Cuaderno de campo. M. Fernández Enguita. ¿Tecnología para crecer o para entretenernos? La brecha de uso de las nuevas tecnologías en la escuela española. Disponible: http://blog.enguita.info/2018/03/tecnologia-para-crecer-o-para.html

Digital Life in 2025. Disponible:

http://www.pewinternet.org/files/2014/03/PIP_Report_Future_of_the_Internet_Predictions_0311 14.pdf

Designing the future of classroom (Innovative Technologies for Engaging Classrooms). Disponible: http://itec.eun.org, http://fcl.eun.org/about, http://envisioning.io/education/

Innovación educativa. Serie "Herramientas de apoyo para el trabajo docente". Texto 1 (UNESCO oficina de Perú, marzo 2016). Disponible: http://docentesinnovadores.perueduca.pe/wpcontent/uploads/2017/05/UNESCO INNOVACI\%C3\%93N.pdf

La integración de las TIC y los libros de texto digitales en la educación. Disponible: http://www.aulaplaneta.com/descargas/aulaPlaneta_Dossier-estudio-TIC.pdf

NMC Horizon Repot: 2013 k-12. Disponible: https://www.nmc.org/nmc-horizon/

Red Educativa Escolar (Tiching). Disponible: http://www.tiching.com/

YoungMarketing. Disponible: http://www.youngmarketing.co/los-11-colegios-mas-innovadores-delmundo/ 